\title{
Simulation of the Pion Decay Channel of a Neutrino Factory
}

\author{
E. B. Holzer
}

\begin{abstract}
In the pion decay channel of a neutrino factory the particles are transported in a solenoidal magnetic field, a 1.8 T constant field in the CERN reference scenario. Increasing the field strength decreases the transverse emittance of the decay muons. To define a lower limit on the achievable $\mu$ emittance, the reference scenario is compared with a study case where pions decay in a field of $20 \mathrm{~T}$. This simulation shows that in any realistic scenario the $\mu$ emittance will only be reduced by less than $21 \%$ in each transverse plane as compared to the reference scenario, and at the expense of an increase in longitudinal emittance.
\end{abstract}

Presented at NUFACT'01, Tsukuba, Japan

25-30 May 2001

Geneva, Switzerland

August 2001 
In the CERN reference scenario for a neutrino factory $[3,4]$ intense beams of electron and muon neutrinos are produced by high energy muons decaying in the long straight sections of a muon decay ring. Those muons in turn are decay products of pions which are produced by a $4 \mathrm{MW}$ beam of protons on a liquid mercury target. For pion capture, the target is inserted either in a magnetic horn or a $20 \mathrm{~T}$ solenoid. This is followed by the long $(30 \mathrm{~m})$ decay channel.

The pion production in the target, which serves as input distribution for this decay channel study, was simulated with the computer code MARS [1] for $10^{6}$ protons at $2.2 \mathrm{GeV}$ hitting the target instantaneously. The target is $26 \mathrm{~cm}$ long, has a radius of $0.75 \mathrm{~cm}$ and is tilted by $50 \mathrm{mrad}$ with respect to the magnetic axis of a $20 \mathrm{~T}$ solenoid. The bore radius of the solenoid is $7.5 \mathrm{~cm}$ and hence pions with a transverse momentum, $p_{T}$, of less than about $250 \mathrm{MeV} / \mathrm{c}$ are captured. In the simulation, $44.5 \times 10^{3} \pi^{+}$and $1.2 \times 10^{3} \mu^{+}$are produced in the forward direction. Of those $15.5 \times 10^{3} \pi^{+}$and $0.1 \times 10^{3} \mu^{+}$fall into the longitudinal momentum range of $180 \mathrm{MeV} / \mathrm{c}$ $<p_{z}<450 \mathrm{MeV} / \mathrm{c}$. The $20 \mathrm{~T}$ field is tapered to $1.8 \mathrm{~T}$ over a distance of 2.02 meter according to $B_{z}(z)=\frac{B_{0}}{1+\alpha \cdot z}$ at $r=0$, where $\alpha=5 \mathrm{~m}^{-1}$. The following $31 \mathrm{~m}$ decay channel has a radius of $30 \mathrm{~cm}$ and a constant field of $1.8 \mathrm{~T}$. The computer code ICOOL [2] was used to track the pions and muons from the end of the target to the end of the decay channel. Muon decay was switched off in these simulations.

\section{Emittance Increase during the Decay Section and the Study Case}

When pions decay to muons the transverse and longitudinal emittance increases. The amount of transverse emittance increase depends on the focusing of the channel. The effect of the additional divergence due to decay on the transverse emittance is smaller for bigger initial divergence of the pions and therefore higher $B_{z}$. The aim of this simulation is to define the achievable minimum transverse muon emittance. There are a number of other influences on the emittance. During a drift (or propagation in a constant longitudinal B-field) the longitudinal rms emittance increases due to weak relativistic effects. The longitudinal emittance of a highly divergent relativistic beam increases during drift (or propagation) because of the dependence of $\gamma$, the relativistic gamma factor, on the transverse velocity, $v_{T}$. The tapered solenoid transforms transverse momentum into longitudinal momentum, ideally without increasing the 6D emittance.

To quantify the correlation between the muon emittance and the B-field in the decay channel, an extreme scenario is chosen for comparison with the reference scenario. The pions decay in a long $20 \mathrm{~T}$ solenoid field, and tapering of the field from 20 to $1.8 \mathrm{~T}$ takes place 30 $\mathrm{m}$ after the target, rather than in the first $2 \mathrm{~m}$. All realistic decay channel designs will have a lower magnetic field, hence this channel defines a lower limit for the achievable transverse muon emittance.

\section{Results and Discussion}

The transverse and longitudinal phase space of muons at the end of the decay channel is shown in figure 1 . On the left is the reference scenario and the $20 \mathrm{~T}$ decay channel is on the right. Transversally an acceptance of $2.27 \pi \mathrm{cm}$ rad (unnormalized) is indicated. It corresponds to $6 \pi$ $\mathrm{cm}$ rad normalized which is 4 times the acceptance of the recirculating accelerator downstream. (The CERN reference scenario foresees a reduction of $\epsilon_{T}$ by a factor of 4 in both planes, [5, 6].) Longitudinally, muons with a $p_{z}$ in the range of 175 to $390 \mathrm{MeV} / \mathrm{c}$ are shown. Indicated is a cut on a time interval of $11.4 \mathrm{~ns}$, half an RF period of $44 \mathrm{MHz}$ (corresponding to the RF of the phase rotation and first muon cooling section $[5,6])$. 

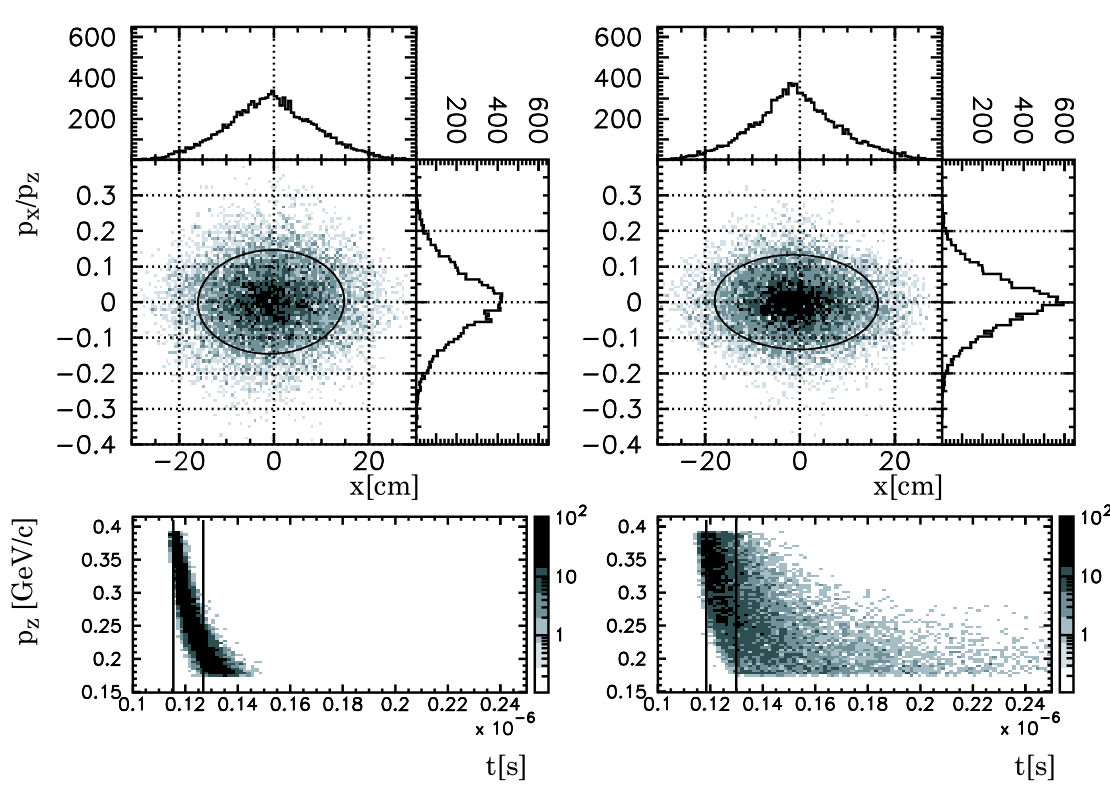

Figure 1: Transverse and longitudinal phase space of muons after pion decay in a field of $1.8 \mathrm{~T}$ (left) and $20 \mathrm{~T}$ (right).

Table 1 summarizes the normalized rms emittances of pions with $180 \mathrm{MeV} / \mathrm{c}<p_{z}<450$ $\mathrm{MeV} / \mathrm{c}, 10 \mathrm{~cm}$ after the target and of the decay muons from these pions $33 \mathrm{~m}$ after the target. The transverse rms emittance increase due to decay is $40.5 \%$ in the $1.8 \mathrm{~T}$ case and $11.1 \%$ in the $20 \mathrm{~T}$ case in both planes. The longitudinal rms emittance is 4.8 times bigger in the $20 \mathrm{~T}$ case than in the reference scenario.

The number of muons $33 \mathrm{~m}$ after the target within the transverse acceptance of $6 \pi \mathrm{cm}$ $\mathrm{rad}$, in the range of $175 \mathrm{MeV} / \mathrm{c}<p_{z}<390 \mathrm{MeV} / \mathrm{c}$ and a time interval of $11.4 \mathrm{~ns}$ is $4.7 \times 10^{3}$ for a decay channel at $1.8 \mathrm{~T}$ and $3.9 \times 10^{3}$ for a decay channel at $20 \mathrm{~T}$.

\begin{tabular}{|l|c|c|c|c|}
\hline$\epsilon[\pi \mathrm{cm} \mathrm{rad}]$ & $\epsilon_{x}$ & $\epsilon_{y}$ & $\epsilon_{L}$ & $\epsilon_{x} \cdot \epsilon_{y} \cdot \epsilon_{L}$ \\
\hline$\pi(10 \mathrm{~cm}$ after target $)$ & 1.74 & 1.69 & 1.3 & 3.8 \\
\hline$\mu($ decayed at $1.8 \mathrm{~T})$ & 2.38 & 2.44 & 49.4 & 287 \\
\hline$\mu($ decayed at $20 \mathrm{~T})$ & 1.91 & 1.90 & 237 & 860 \\
\hline
\end{tabular}

Table 1: Summary of normalized rms emittances. Pions are at $10 \mathrm{~cm}$ after the target and muons at the end of the decay channel (33 $\mathrm{m}$ after the target). Both channels have the same transmission.

The difference in longitudinal phase space in the two scenarios mainly originates from the travel time difference, $\Delta t$, over the same distance between two pions with the same $p_{z}$ and a $p_{T}$ of zero and maximum transverse momentum, $p_{T, \max }$, respectively:

$$
\Delta t=\left.t\right|_{p_{T}=0} \cdot\left(\sqrt{1+\frac{p_{T, \max }^{2}}{\left.E^{2}\right|_{p_{T}=0}}}-1\right)
$$

Where $\left.t\right|_{p_{T}=0}$ and $\left.E\right|_{p_{T}=0}$ are the flight time and energy of a pion with $p_{T}=0$, and $p_{T, \max }$ is a function of the B-field. In the approximation of adiabatic B-field changes, the invariance of the action integral leads to $B / p_{T}^{2}=$ const. The geometry at the target gives $p_{T, \max }[\mathrm{MeV} / \mathrm{c}] \simeq$ 


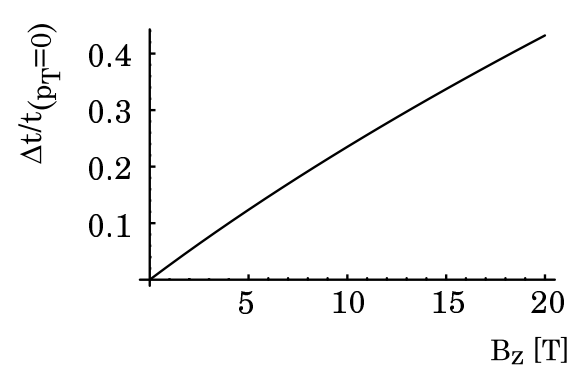

Figure 2: Relative travel time difference over a distance of $30 \mathrm{~m}$ between pions of the same $p_{z}$ as a function of $B$.

$55.9 \cdot \sqrt{B_{z}[T]}$. Figure 2 shows $\Delta t /\left.t\right|_{p_{T}=0}$ as a function of $B_{z}$ for pions with a $p_{z}$ of $200 \mathrm{MeV} / \mathrm{c}$ in this approximation. The function is nearly linear in the range of $0 \mathrm{~T}<B_{z}<20 \mathrm{~T}$ and reaches $43 \%$ at $20 \mathrm{~T}$.

\section{Conclusions}

Rms emittances and the number of muons in a given acceptance (longitudinal acceptance of the first cooling section and 4 times the transverse acceptance of the recirculating linac) have been compared for the reference cooling channel and an extreme scenario. Higher B-fields decrease the transverse and increase the longitudinal emittance. In any realistic scenario (with a B-field less than $20 \mathrm{~T}$ ) the transverse emittance will be decreased by less than $21 \%$ in each plane as compared to the reference scenario. The final choice of $B_{z}$ will depend on the performance and acceptance of the following elements for cooling and acceleration. The number of muons in a given acceptance is higher for the lower value of $B_{z}$, but further simulations are necessary to determine the position of the maximum.

\section{References}

[1] S. Gilardoni, Review and comparison of solenoid and horn capture scheme, to be published.

[2] R.C. Fernow, ICOOL: A simulation code for ionization cooling of muon beams, Proc. 1999 Particle Accelerator Conference, New York (1999).

[3] H. Haseroth for the CERN Neutrino Factory Working Group, CERN Ideas and Plans for a Neutrino Factory, submitted to NIMPR-A, CERN/PS 2000-064 (PP).

[4] R. Garoby for the Neutrino Factory Working Group, Current Activities for a Neutrino Factory at CERN, HEACC'2001, CERN/PS 2001-007 (RF).

[5] G. Franchetti et al., Phase Rotation, Cooling and Acceleration of Muon Beams: a Comparison of different Approaches, submitted to NIMPR-A, CERN/PS 2000-054.

[6] A. Lombardi, A 40-80 MHz System for Phase Rotation and Cooling, CERN-NUFACT note 34 (2000). 